\title{
Calculating Four-Loop Corrections in QCD
}

\author{
S. Moch and V. Magerya
}

\begin{abstract}
We review the current status of perturbative corrections in QCD at four loops for scattering processes with space- and time-like kinematics at colliders, with specific focus on deep-inelastic scattering and electron-positron annihilation. The calculations build on the parametric reduction of loop and phase space integrals up to four-loop order using computer algebra programs such as Form, designed for large scale computations.
\end{abstract}

\section{Introduction}

Perturbation theory forms the backbone of theory predictions for scattering processes at high energy colliders. Given the size of the coupling constant $\alpha_{s}$ in the theory of strong interactions, Quantum Chromodynamics (QCD), this requires the computation of quantum corrections at higher orders. For collisions involving hadrons, either in the initial state or identified in the final state, the theory description is based on QCD factorization, which allows for the separation of long- and short-distance physics. Within this framework, quantum corrections to the hard scattering cross section driven by short-distance physics are calculated typically at the next-to-nextto-leading order (NNLO) in order to reach an accuracy of the order of a few percent from the truncation of the perturbative expansion. The long-distance physics part of the interaction is encoded in hadronic matrix elements which are inaccessible to

\footnotetext{
S. Moch

II. Institut für Theoretische Physik, Universität Hamburg

Luruper Chaussee 149, D-22761 Hamburg, Germany

e-mail: sven-olaf.moch@desy.de

V. Magerya

Institut für Theoretische Physik, Campus Süd

Karlsruher Institut für Technologie (KIT), D-76128 Karlsruhe, Germany

e-mail: vitalii.maheria@kit.edu
} 
perturbation theory. Based on the description of a hadron as an incoherent combination of parton states, it is possible, though, to compute matrix elements of partonic operators and, in particular, their scale dependence in perturbative QCD. The gained knowledge, encoded in splitting functions, serves as input to the parton evolution equations derived from the renormalization group and forms an essential ingredient in the determination of the non-perturbative parton distribution functions (PDFs) or parton fragmentation functions (FFs) from fits to experimental data. The current description of QCD evolution equations for PDFs and FFs is complete at NNLO. This requires the splitting functions in space-like and time-like kinematics at the three-loop level [1, 2, 3, 4, 5, 6] as well as the coefficient functions for the hard scattering at two-loop order entering, e.g., in DIS structure functions [7, 8, 9, 10] or in fragmentation functions in $e^{+} e^{-}$annihilation [11, 12, 13, 3, 14].

With the increasing precision of the experimental data collected at the Large Hadron Collider (LHC) for Standard Model (SM) processes used to extract fundamental theory parameters such as the strong coupling $\alpha_{s}$ or the PDFs [15], the step towards the next-to-next-to-next-to-leading order $\left(\mathrm{N}^{3} \mathrm{LO}\right)$ becomes necessary. This is particularly crucial in preparation for the physics program at a future Electron-Ion Collider (EIC) [16, 17], where PDFs as well as parton FFs are expected to be accessible with high precision, but also in view of the ongoing future circular collider (FCC) studies [18]. The push beyond the state-of-the-art requires the calculation of four-loop corrections, building on known results for the renormalization of QCD at four-loop [19, 20] and even five-loop order [21, 22, 23].

The simplest cross section computations at the four-loop level involve semiinclusive (single-scale) observables, such as DIS structure functions and $e^{+} e^{-}$fragmentation functions and the current status of their calculation will be discussed and reviewed in detail below.

\section{Space-like kinematics}

The scattering reaction for unpolarized DIS reads

$$
l(k)+\operatorname{nucl}(p) \rightarrow l^{\prime}\left(k^{\prime}\right)+X,
$$

where $l$ and $l$ ' denote the scattered lepton and 'nucl' a nucleon with respective momenta $k, k^{\prime}$ and $p . X$ summarizes the remaining hadronic final states. The inclusive DIS cross section factorizes as $d \sigma \sim L^{\mu \nu} W_{\mu \nu}$ in terms of leptonic and hadronic tensors $L_{\mu \nu}$ and $W_{\mu \nu}$. The latter one encodes the strong interaction dynamics and can be expanded to define the unpolarized structure functions $F_{2,3, L}$,

$$
\begin{aligned}
W_{\mu \nu}(p, q) & =\frac{1}{4 \pi} \int d^{4} z \mathrm{e}^{\mathrm{i} q \cdot z}\left\langle\text { nucl, } \mathrm{p}\left|J_{\mu}^{\dagger}(z) J_{v}(0)\right| \text { nucl, } \mathrm{p}\right\rangle \\
& =\frac{e_{\mu \nu}}{2 x_{B}} F_{L}\left(x_{B}, Q^{2}\right)+\frac{d_{\mu \nu}}{2 x_{B}} F_{2}\left(x_{B}, Q^{2}\right)+\mathrm{i} \frac{\epsilon_{\mu \nu p q}}{p \cdot q} F_{3}\left(x_{B}, Q^{2}\right) .
\end{aligned}
$$


Here $J_{\mu}$ represents an electro-magnetic or weak current. The momentum $q$ is transferred by the gauge-boson with space-like kinematics, $Q^{2} \equiv-q^{2}>0$, and the Bjorken variable is defined as

$$
x_{B}=\frac{Q^{2}}{2 p \cdot q},
$$

with $0<x_{B} \leq 1$. The symmetric tensors $e_{\mu \nu}$ and $d_{\mu \nu}$ multiplying the structure functions $F_{2, L}$ are dependent on $p$ and $q$, while the totally antisymmetric one $\epsilon_{\mu v \alpha \beta}$ in front of the structure function $F_{3}$ arises from the vector/axial-vector interference, see [24, 25] for definitions.

QCD factorization allows for the decomposition of the DIS structure functions in terms of (space-like) coefficient functions $C_{a, \mathrm{f}}$ and PDFs $\phi_{\mathrm{f}}$,

$$
F_{a}\left(x_{B}, Q^{2}\right)=\sum_{\mathrm{f}=\mathrm{q}, \overline{\mathrm{q}}, \mathrm{g}} \int_{x_{B}}^{1} \frac{d z}{z} \phi_{\mathrm{f}}\left(\frac{x_{B}}{z}, \mu^{2}\right) C_{a, \mathrm{f}}\left(z, \alpha_{\mathrm{s}}\left(\mu^{2}\right), \frac{\mu^{2}}{Q^{2}}\right)+O\left(\frac{1}{Q^{2}}\right),
$$

up to higher-twist corrections $O\left(1 / Q^{2}\right)$. The coefficient functions can be computed in perturbation theory via expansions in the strong coupling $a_{\mathrm{s}} \equiv \alpha_{\mathrm{s}} /(4 \pi)$ as

$$
C_{a, \mathrm{f}}\left(x, \alpha_{\mathrm{s}}\right)=\delta(1-x)+a_{\mathrm{s}} c_{a, \mathrm{f}}^{(1)}(x)+a_{\mathrm{s}}^{2} c_{a, \mathrm{f}}^{(2)}(x)+a_{\mathrm{s}}^{3} c_{a, \mathrm{f}}^{(3)}(x)+a_{\mathrm{s}}^{4} c_{a, \mathrm{f}}^{(4)}(x)+\ldots,
$$

and are completely known up to $\mathrm{N}^{3} \mathrm{LO}$ [24, 25], i.e. all terms $c_{a, \mathrm{f}}^{(3)}$. At four-loop order a low number of fixed Mellin moments, defined as

$$
c(N)=\int_{0}^{1} d x x^{N-1} c(x),
$$

are available [26] as well as the complete soft corrections in the limit $x \rightarrow 1$ using threshold resummation and QCD factorization in $d$-dimensions [27].

The scale dependence of the PDFs is governed by the well-known evolution equations

$$
\frac{d}{d \ln \mu^{2}} \phi_{\mathrm{f}}=\sum_{\mathrm{f}^{\prime}=\mathrm{q}, \overline{\mathrm{q}}, \mathrm{g}} P_{\mathrm{ff}^{\prime}}\left(\alpha_{s}\left(\mu^{2}\right)\right) \otimes \phi_{\mathrm{f}^{\prime}}\left(x, \mu^{2}\right) .
$$

For QCD with $n_{f}$ quark flavors and with ' $\otimes$ ' denoting the standard convolution these are commonly expressed in terms of $2 n_{f}-1$ scalar equations in the flavor non-singlet case, and a coupled set of $2 \times 2$ matrix equations in the flavor singlet case. The evolution kernels, i.e. the space-like splitting functions $P_{\mathrm{ff}}$ are calculable in perturbative QCD as well,

$$
P_{\mathrm{ff}^{\prime}}\left(x, \alpha_{\mathrm{s}}\right)=a_{\mathrm{s}} P_{\mathrm{ff}^{\prime}}^{(0)}(x)+a_{\mathrm{s}}^{2} P_{\mathrm{ff} f^{\prime}}^{(1)}(x)+a_{\mathrm{s}}^{3} P_{\mathrm{ff}^{\prime}}^{(2)}(x)+a_{\mathrm{s}}^{4} P_{\mathrm{ff}^{\prime}}^{(3)}(x)+a_{\mathrm{s}}^{5} P_{\mathrm{ff} f^{\prime}}^{(4)}(x)+\ldots .
$$


The NNLO results $P_{\mathrm{ff}}^{(2)}$ are all known [1, 2]. At $\mathrm{N}^{3} \mathrm{LO}$, i.e., at four loops, the nonsinglet quark-quark splitting functions have been computed in the large- $N_{c}$ limit and number of Mellin moments for the remaining color coefficients are known [28, 29] for a general $S U\left(N_{c}\right)$ gauge theory. In the flavor-singlet sector the leading large- $n_{f}$ terms and those proportional to quartic color Casimirs are known [30, 29]. Beyond this order, even some low- $N$ Mellin moments of the five-loop contributions to the non-singlet quark-quark splitting function $P_{\mathrm{ns}}^{(4)}$ have been determined [31].

In the following, we give a brief overview of the computational set-up and workflow underlying the computations at four loops and beyond.

\subsection{Computational work-flow}

Using the operator product expansion in DIS one can relate the product of currents $J_{\mu}$ in the hadronic tensor in Eq. (2) to Mellin moments of the structure functions $F_{2,3, L}$, see, e.g. [32]. The latter, parameterizing the (semi)-inclusive cross section, are then obtained with the help of the optical theorem from the imaginary part of the forward Compton amplitude for the gauge boson-nucleon scattering. Thus, the computation of QCD corrections in DIS starts from the forward Compton amplitude of the corresponding gauge boson-parton scattering process, using the kinematics of Eq. (3), which gives access to both, the coefficient functions in Eq. (5) and the splitting functions in Eq. (8). In case, one is only interested in the latter, the direct computation of operator matrix elements, evaluated in parton two-point functions, proves more efficient and allows for the determination of the anomalous dimensions $\gamma(N)$, i.e., the Mellin transforms of the splitting functions, cf. Eq. (6).

The required Feynman diagrams up to four loops can generated using the diagram generator Qgraf [34] and the group theory factors for a general color $S U\left(N_{c}\right)$ gauge theory can be obtained with algorithms described in [35]. The loop integrals are considered in dimensional regularization [36, 37], $d=4-2 \varepsilon$, which is the standard framework in perturbative QCD at higher orders and the integral reductions are performed by means of integration-by-parts identities (IBP) [38, 39]. The solution of the IBP reductions are encoded in the program Forcer [40], which performs a parametric reduction of four-loop massless propagator diagrams to master integrals. The latter are shown in Fig. 1 and their analytic expressions as a Laurent series in $\varepsilon$ have been computed in Refs. [41, 42]. The symbolic manipulations employ the computer algebra system Form [43, 44, 45] and its multi-threaded version TForm [46] in order to handle both, the run times and the size of the intermediate expressions occurring in the reduction of diagrams with high Mellin moments $N$.

The approach delivers results for fixed Mellin moments of the anomalous dimensions and DIS coefficient functions. When enough fixed Mellin moments are available, one can follow the approach of [47], and attempt the reconstruction of an analytic expression as a function of $N$ in terms of harmonic sums [48, 49]. In the planar limit, i.e., for large $N_{c}$, the exact four-loop results for moments up to $N=20$ are sufficient to determine the analytic expressions of the non-singlet 


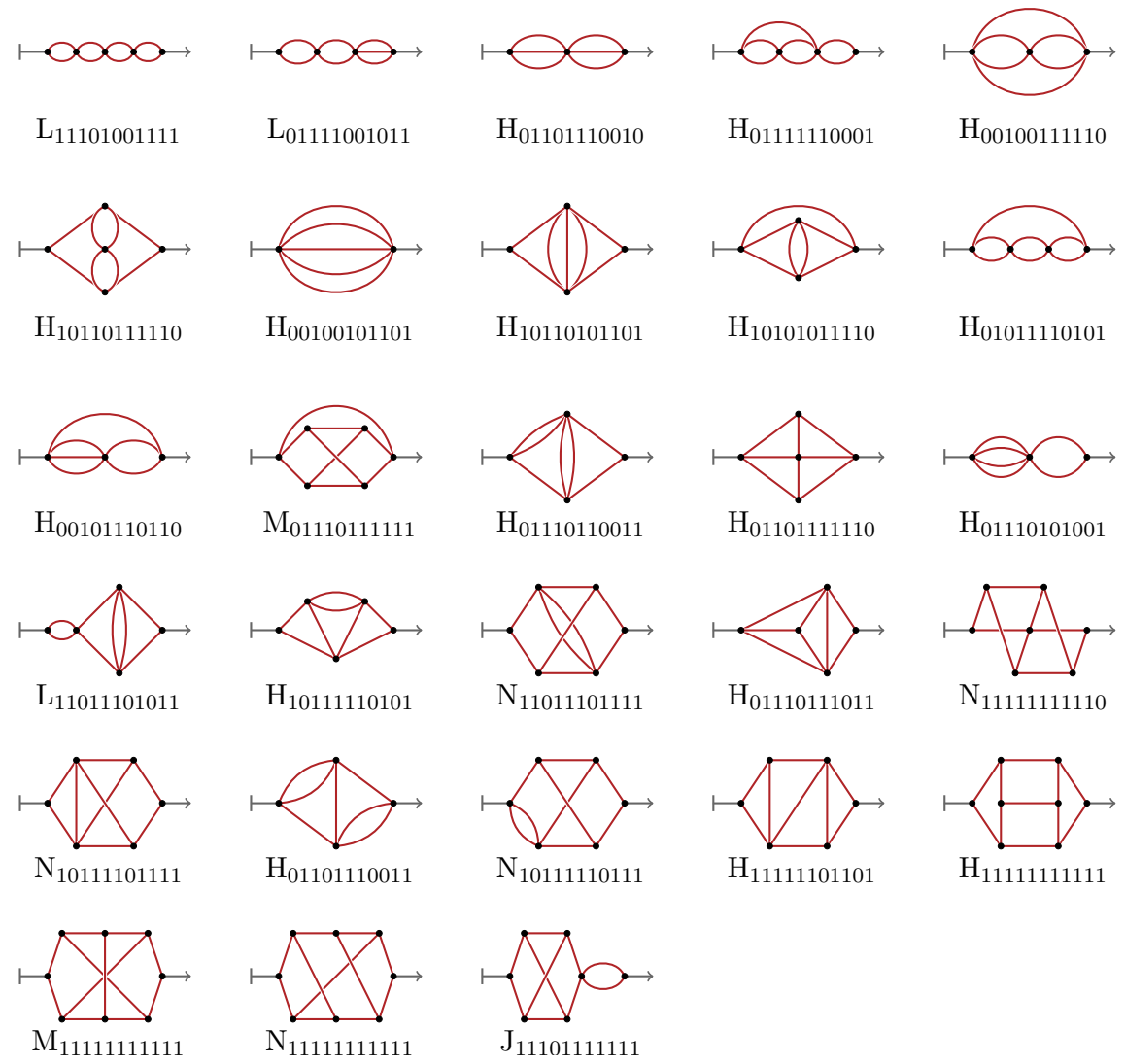

Fig. 1 Master integrals for four-loop propagators (figure from [33]).

quark-quark anomalous dimensions $\gamma_{\mathrm{ns}}^{(3)}(N)$ as a function of $N$ by LLL-based techniques [50, 51, 52, 53] and solving systems of Diophantine equations, cf. [28] for details.

The bottleneck of the approach via fixed Mellin moments is caused by the high powers of propagators, which need to undergo the parametric reduction with the program Forcer [40]. This leads to large intermediate expressions of the order of TByte and to long run times of the computer algebra system Form. For example, the computation of the Mellin moment $N=10$ of the quark coefficient function in the projection on $F_{L}$ in Eq. (4) at four loops requires the evaluation $O(3200)$ diagrams with a total of $O(800000)$ hrs CPU time, i.e. almost 100 years altogether. Fortunately, the multi-threaded version TForm delivers an average speed-up factor of $O(10)$ and with a cluster of sufficiently many servers, the problem is doable within half a year of "wall time".

Extensions to five-loop low- $N$ Mellin moments of the non-singlet anomalous dimension $\gamma_{\mathrm{ns}}^{(4)}(N)$, i.e. the Mellin transform of $P_{\mathrm{ns}}^{(4)}$, as achieved in [31], require 
the computation of five-loop self-energy integrals, which can be accomplished with an implementation [54] of the local $\mathrm{R}^{*}$ operation [55, 56, 57]. This allows for the reduction to four-loop integrals, that can be evaluated again by the Forcer program [40]. However, the size of intermediate expressions and the run times of Form become prohibitively large beyond the fixed values $N=2$ and $N=3$.

\section{Time-like kinematics}

Semi-inclusive $e^{+} e^{-}$annihilation via a virtual photon or Z-boson with time-like momentum $q$ proceeds as

$$
e^{-}+e^{+} \rightarrow \gamma / Z(q) \rightarrow h(p)+X
$$

where $h(p)$ stands for a specific species of identified hadrons in the final state. The time-like kinematics are characterized by the momentum transfer $Q^{2} \equiv q^{2}>0$ and the Feynman variable is

$$
x_{F}=\frac{2 p \cdot q}{Q^{2}},
$$

with $0<x_{F} \leq 1$. In the center-of-mass frame $x_{F}$ is the fraction of the beam energy carried by the hadron $h$. The space- and time-like processes (1) and (9) are related by crossing which implies a mapping $x_{B} \rightarrow x_{F}$ for the kinematics in Eqs. (3) and (10) and the use of analytic continuation.

In perturbative QCD, the total (angle-integrated) fragmentation function

$$
\frac{1}{\sigma_{\mathrm{tot}}} \frac{d \sigma^{h}}{d x}=F^{h}\left(x, Q^{2}\right),
$$

as well as the transverse $\left(F_{T}^{h}\right)$, longitudinal $\left(F_{L}^{h}\right)$ and asymmetric $\left(F_{A}^{h}\right)$ ones parameterizing the double-differential cross section $d \sigma^{h} / d x d \cos \theta_{h}$ [58], are given by

$$
F_{a}^{h}\left(x, Q^{2}\right)=\sum_{\mathrm{f}=\mathrm{q}, \overline{\mathrm{q}}, \mathrm{g}} \int_{x}^{1} \frac{d z}{z} C_{a, \mathrm{f}}^{T}\left(z, \alpha_{\mathrm{s}}\left(Q^{2}\right)\right) D_{\mathrm{f}}^{h}\left(\frac{x}{z}, Q^{2}\right)+O\left(\frac{1}{Q}\right),
$$

in terms of the parton fragmentation functions (FFs) $D_{\mathrm{f}}^{h}$ and the (time-like) coefficient functions $C_{a, \mathrm{f}}^{T}$,

$$
C_{a, \mathrm{f}}^{T}\left(x, \alpha_{\mathrm{s}}\right)=\sigma_{\mathrm{ew}}\left(\delta(1-x)+a_{\mathrm{s}} c_{a, \mathrm{f}}^{T,(1)}(x)+a_{\mathrm{s}}^{2} c_{a, \mathrm{f}}^{T,(2)}(x)+a_{\mathrm{s}}^{3} c_{a, \mathrm{f}}^{T,(3)}(x)+\ldots\right),
$$

where $\sigma_{\text {ew }}$ denotes the electroweak pre-factors [58] and the second-order coefficient functions are known [11, 12, 13, 3, 14], while the three-loop corrections $c_{a}^{T,(3)}(x)$ have not been derived so far. 
The parton FFs $D_{\mathrm{f}}^{h}$ obey evolution equations analogous to the PDFs in Eq. (7),

$$
\frac{d}{d \ln \mu^{2}} D_{\mathrm{f}}^{h}=\sum_{\mathrm{f}^{\prime}=\mathrm{q}, \overline{\mathrm{q}}, \mathrm{g}} P_{\mathrm{f}^{\prime} \mathrm{f}}^{T}\left(\alpha_{s}\left(\mu^{2}\right)\right) \otimes D_{\mathrm{f}^{\prime}}^{h}\left(x, \mu^{2}\right),
$$

with time-like splitting functions, but with $2 \times 2$ matrix $P_{f^{\prime} f}^{T}$ in the flavor singlet case transposed compared to PDFs in Eq. (7). In perturbative QCD the time-like splitting functions can be expanded as

$$
P_{\mathrm{f}^{\prime} \mathrm{f}}^{T}\left(x, \alpha_{\mathrm{s}}\right)=a_{\mathrm{s}} P_{\mathrm{f}^{\prime} \mathrm{f}}^{T,(0)}(x)+a_{\mathrm{s}}^{2} P_{\mathrm{f}^{\prime} \mathrm{f}}^{T,(1)}(x)+a_{\mathrm{s}}^{3} P_{\mathrm{f}^{\prime} \mathrm{f}}^{T,(2)}(x)+a_{\mathrm{s}}^{4} P_{\mathrm{f}^{\prime} \mathrm{f}}^{T,(3)}(x)+\ldots,
$$

where the NNLO results $P_{\mathrm{f}, \mathrm{f}}^{T,(2)}$ are all known [3, 4, 5, 6], while at $\mathrm{N}^{3} \mathrm{LO}$ only the nonsinglet quark-quark splitting functions are available in the large- $N_{c}$ limit [28]. These results are all based on analytic continuation from space- to time-like kinematics and on exploiting reciprocity relations for collinear splitting functions in QCD. In the sequel, we discuss the computational work-flow for the direct calculation of QCD corrections to semi-inclusive $e^{+} e^{-}$annihilation (9).

\subsection{Inclusive cross-sections}

A practical indirect way of calculating total cross-sections is the optical theorem. Through it, $O\left(\alpha_{s}^{3}\right)$ corrections for $e^{+} e^{-}$annihilation in Eq. (9) can be expressed in terms of the four-loop propagator diagrams. In the massless case all the 22 master integrals for these propagators shown in Fig. 1 1 have been calculated in [41, 42].

The direct way on the other hand requires the calculation of all squared amplitudes with $2,3,4$, and 5 particles in the final state (with 3,2, 1, and 0 loops respectively), e.g.

$$
\sigma \sim \sum_{n} \int \mathrm{dPS}_{n}\left|\left\langle p_{1}, \ldots, p_{n}|i T| q\right\rangle\right|^{2}=\int \mathrm{dPS}_{3} \mid
$$

and integration of those over the respective phase space,

$$
\mathrm{dPS}_{n} \equiv\left(\prod_{i=1}^{n} \frac{\mathrm{d}^{d} p_{i}}{(2 \pi)^{d-1}} \delta^{+}\left(p_{i}^{2}\right)\right)(2 \pi)^{d} \delta^{d}\left(q-\sum_{j=1}^{n} p_{j}\right) .
$$

Performing the phase space integration analytically quickly turns out to be the bottleneck: the parameterization of 4- and 5- particle phase spaces necessarily requires the introduction of square roots into the integrand, preventing an analytic solution, see, e.g., the "tripole parameterization" of the 4-particle phase space in [59]. Instead one should consider both loop and phase space integration appearing in the squared amplitude together, as a single "cut" diagram: 


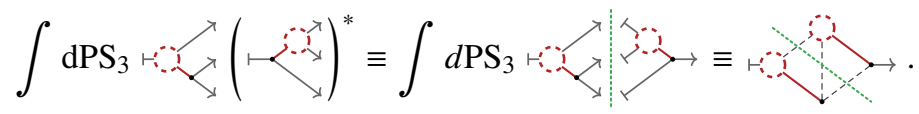

Then, by applying the idea of "reverse unitarity" [60]: that is, replacing on-shell conditions for final state particles in Eq. (17) by denominators,

$$
\delta^{+}\left(p^{2}\right)=\frac{1}{2 \pi i}\left(\frac{1}{p^{2}-i 0}-\frac{1}{p^{2}+i 0}\right),
$$

one can treat each outgoing line as a "cut propagator", and thus construct IBP relations for cut diagrams.

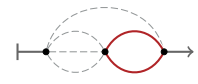

$\mathrm{H}_{00 * 01 * * 0 * 10}$

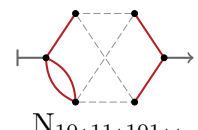

$\mathrm{N}_{10 * 11 * 101 * *}$
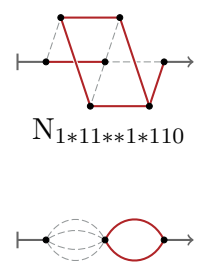

$\mathrm{H}_{0 * 110 * 0 * 00 *}$

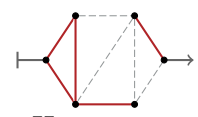

$\mathrm{H}_{11 * 1 * 101 * 0 *}$
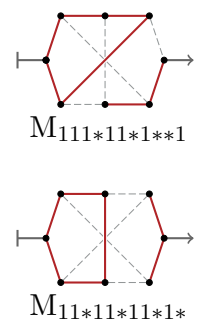
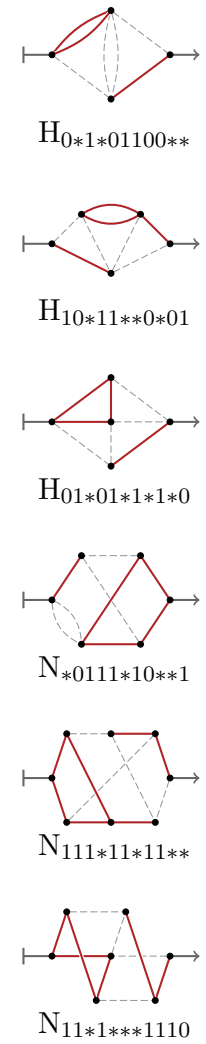

$\mathrm{H}_{0 * 1 * 01100 * *}$

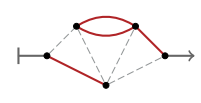

$\mathrm{H}_{10 * 11 * * 0 * 01}$
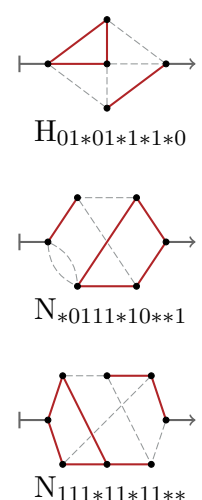

$\mathrm{N}_{11 * 1 * * * 1110}$

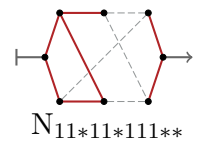

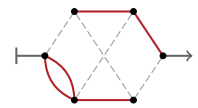

$\mathrm{N}_{101 * 11 * 01 * *}$
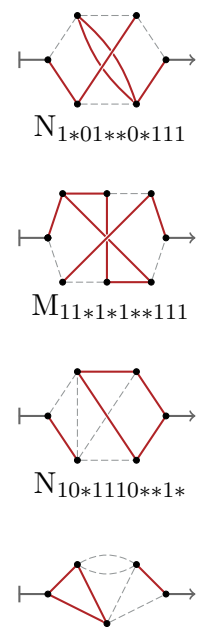

$\mathrm{H}_{10 * 1 * 110 * 0 *}$
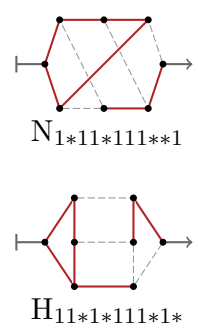

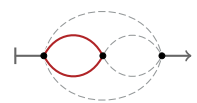

$\mathrm{H}_{00 * 00 * 1 * 1 * 0}$

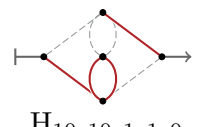

$\mathrm{H}_{10 * 10 * 1 * 1 * 0}$
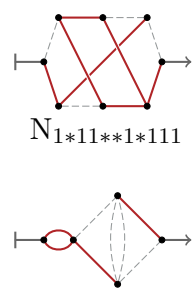

$\mathrm{L}_{1 * 0 * * * 01011}$

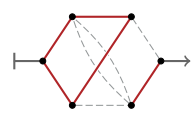

$\mathrm{N}_{1 * 01 * 101 * * 1}$
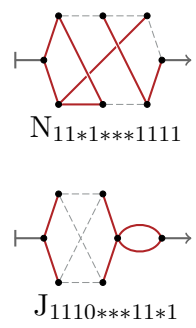

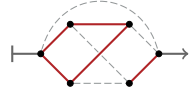

$\mathrm{M}_{011 * 01 * 1 * * 1}$
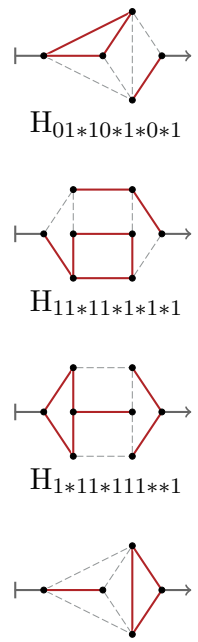

$\mathrm{H}_{0 * 110 * 1 * 01 *}$
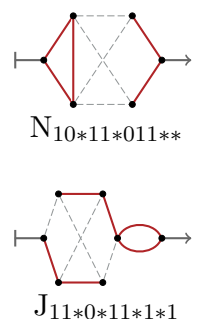

Fig. 2 Master integrals for all four-particle cuts of four-loop propagators (figure from [33]). 
In this way the direct calculation is reduced to calculating the master integrals for 2-, 3-, 4-, and 5- particle cuts of four-loop propagators. For this task conventional IBP software can be largely reused with two modifications: first, any cut propagator raised to a non-negative power can be set to zero (because $x \delta(x)=0$ ), and second, when symmetries between diagrams are constructed, cut propagators should not be symmetrized with the regular ones.

The full set of (massless) master integrals for 5-particle cuts of four-loop propagators has been first calculated in [61], for 4- and 3-particle cuts in [33], and for 2-particle cuts in [62, 63, 64]. As an example, the set of master integrals for 4-particle cuts is shown in Fig. 2. Because these integrals are single-scale, it is convenient to calculate them by solving dimensional recurrence relations (DRR) [65, 66], which relate the values of these integrals at different values of the space-time dimension $d$ :

$$
I_{i}(d+2)=M_{i i} I_{i}(d)+\sum_{j \neq i} M_{i j} I_{j}(d) .
$$

As explained in [33], there is at most a single master integral per sector, and thus the matrix $M_{i j}$ is triangular. With the help of the "dimensional recurrence and analyticity" method of [67], an ansatz for the full solution can be constructed, leaving only a number of constants undetermined. Once enough extra information is gathered to fix these constants (i.e. values of the leading pole coefficients, or several terms of the $\varepsilon$-expansion computed by alternative means), DREAM [68] can be used to evaluate $I_{i}(4-2 \varepsilon)$ as a series in $\varepsilon$ with arbitrary precision (thousands of digits), and these numerical values can then be turned into analytic expressions in terms of multiple zeta values [69] with the help of an integer relation reconstruction algorithm like PSQL [70].

The optical theorem (or rather Cutkosky rules [71, 72]), being the alternative way of computing fully inclusive quantities, provides an essential cross-check on these master integrals: the imaginary part of each four-loop propagator diagram must be equal to a combination of its cuts. For example:

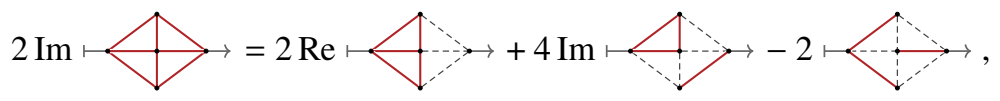

where the dashed lines indicate the propagators cut according to Eq. (19), see also Fig. 2 .

\subsection{Semi-inclusive cross-sections}

Integrals for semi-inclusive cross-sections differ from the inclusive case by the presence of the scaling parameter $x$ (i.e., $x_{F}$ in Eq. (10) ) in the integration measure,

$$
\operatorname{dPS}_{n}(x) \equiv \operatorname{dPS}_{n} \delta\left(x-2 q \cdot p_{1} / q^{2}\right),
$$


so that the semi-inclusive cut diagrams now have the form of

$$
\int \mathrm{dPS}_{3}(x)
$$

where the crossed dashed line corresponds to the constraint in Eq. (22). The inclusion of $x$ still allows for an IBP reduction if one applies Eq. (19) to transform this additional $\delta$-function into a cut propagator, this time a massive one. This complicates calculations:

- first, by introducing linear dependencies between denominators of a given diagram; these need to be split through partial fractioning, with the end result that a single Feynman diagram can now contribute terms to several different meta topologies;

- second, by the increased number of master integrals: there are 693 semi-inclusive master integrals ( 298 for 5-particle cuts, 277 for 4-particle cuts, 96 for 3-particle cuts, and 22 for 2-particle cuts) vs. 115 for the inclusive case;

- third, by the increase in the size of IBP expressions, and the increased computational requirements of the reduction;

- and finally by the fact that one can not easily solve DRR for these integrals: the method used for the inclusive case largely relied on the numerical evaluation, and having a free parameter $x$ makes that impractical (if not impossible).

The master integrals for semi-inclusive cuts for three-loop propagators were completed in [73, 74] and for four-loop propagators in [75].

A convenient way to calculate the values of these master integrals is the method of differential equations [76, 77]: the integrands of cut master integrals can be differentiated with respect to $x$, and the obtained expressions can then be reduced back to the same integrals via IBP relations, resulting in systems of differential equations of the form

$$
\frac{\partial}{\partial x} I_{i}(d, x)=M_{i j}(d, x) I_{j}(d, x)
$$

To solve these equations, one follows the observation from [78]: if there is a basis transformation

$$
J_{i}(d, x)=T_{i j}(d, x) I_{j}(d, x),
$$

such that once substituted into Eq. (24) factorizes the dependence of $M$ on $d=4-2 \varepsilon$, transforming the equation into an $\varepsilon$-form,

$$
\frac{\partial}{\partial x} J_{i}(d, x)=\varepsilon S_{i j}(x) J_{j}(d, x),
$$

then the solution can easily found as a series in $\varepsilon$,

$$
I_{i}(4-2 \varepsilon, x) \equiv \sum_{k} \varepsilon^{k} I_{i}^{k}(x), \quad I_{i}^{(k)}(x)=\int \mathrm{d} x S_{i j}(x) I_{j}^{(k-1)}(x)+C_{i}^{(k)} .
$$


Only two issues remain: how to find the transformation matrix $T_{i j}$ from Eq. 25], and how to fix the integration constants $C_{i}^{(k)}$.

A general algorithm of constructing $\varepsilon$-form transformations directly from the matrix $M_{i j}$ was presented in [79] and improved upon in [18, 80]. We rely upon the public implementation of this algorithm, Fuchsia [81, 82], to find $T_{i j}$, specifically on the new version available at [83].

To fix the integration constants observe that if one integrates a semi-inclusive integrals over all $x$, the result should be a fully inclusive integral. So by writing down equations of the form

$$
\int \mathrm{d} x
$$

for each master integral, and inserting the series' in $\varepsilon$ for both the semi-inclusive and the (known) inclusive integrals, all $C_{i}^{(k)}$ can be recovered. The only complication here is that the solution for $I_{i}(d, x)$ may contain terms $\sim 1 / x$, which would make the integral on the left-hand side divergent if taken order-by-order in the series. This can be side-stepped by multiplying the integrand on the left-hand side by $x^{m}$ with high enough $m$, and inserting a denominator of the form $\left(2 q \cdot p_{1} / q^{2}\right)^{m}$ into the diagram on the right-hand side.

At this stage, it remains to apply the IBP reductions for the semi-inclusive case to the Feynman diagrams of the individual parton processes contributing to the semi-inclusive $e^{+} e^{-}$annihilation in Eq. (9). This will check the NNLO results for the time-like splitting functions $P_{\mathrm{f}^{\prime} \mathrm{f}}^{T,(2)}(x)$ in Eq. (15) by a direct computation and determine the hitherto unknown three-loop corrections $c_{a}^{T,(3)}(x)$ in Eq. (13).

\section{Conclusions}

The push towards $\mathrm{N}^{3} \mathrm{LO}$ accuracy in QCD for DIS structure functions or fragmentation functions in $e^{+} e^{-}$annihilation requires calculations at four-loop order. The efforts are realized with a largely automated work-flow for the generation of all Feynman diagrams, the parametric IBP reduction to master integrals of loop and phase space integrals, for the latter after a mapping with "reverse unitarity" to loop integrals with cuts, and the computation of the master integrals with various algorithms, such as DRR or differential equations. The complexity of the computations, i.e., the size of the expressions, the run times for IBP reductions and the algorithms for the solution of master integrals poses challenges to currently available computer algebra programs and requires continuous improvements. We have presented a brief review of the current status, listing available results as well as indicating the needs for future improvements. 


\section{Acknowledgments}

We acknowledge support by Deutsche Forschungsgemeinschaft (DFG) through the

Research Unit FOR 2926, "Next Generation pQCD for Hadron Structure: Preparing for the EIC", project MO 1801/5-1.

\section{References}

1. S. Moch, J. A. M. Vermaseren, and A. Vogt, Nucl. Phys. B 688, 101 (2004), hep-ph/0403192

2. A. Vogt, S. Moch, and J. A. M. Vermaseren, Nucl. Phys. B 691, 129 (2004), hep-ph/0404111

3. A. Mitov, S. Moch, and A. Vogt, Phys. Lett. B 638, 61 (2006), hep-ph/0604053

4. S. Moch and A. Vogt, Phys. Lett. B 659, 290 (2008), 0709.3899.

5. A. A. Almasy, S. Moch, and A. Vogt, Nucl. Phys. B 854, 133 (2012), 1107.2263.

6. H. Chen, T.-Z. Yang, H. X. Zhu, and Y. J. Zhu, Chin. Phys. C 45, 043101 (2021), 2006.10534.

7. W. L. van Neerven and E. B. Zijlstra, Phys. Lett. B 272, 127 (1991).

8. E. B. Zijlstra and W. L. van Neerven, Phys. Lett. B 273, 476 (1991).

9. E. B. Zijlstra and W. L. van Neerven, Nucl. Phys. B 383, 525 (1992).

10. S. Moch and J. A. M. Vermaseren, Nucl. Phys. B 573, 853 (2000), hep-ph/9912355

11. P. J. Rijken and W. L. van Neerven, Phys. Lett. B 386, 422 (1996), hep-ph/9604436

12. P. J. Rijken and W. L. van Neerven, Nucl. Phys. B 487, 233 (1997), hep-ph/9609377

13. P. J. Rijken and W. L. van Neerven, Phys. Lett. B 392, 207 (1997), hep-ph/9609379

14. A. Mitov and S. Moch, Nucl. Phys. B 751, 18 (2006), hep-ph/0604160

15. A. Accardi et al., Eur. Phys. J. C 76, 471 (2016), 1603.08906.

16. D. Boer et al., (2011), 1108.1713.

17. A. Accardi et al., Eur. Phys. J. A 52, 268 (2016), 1212.1701.

18. A. Blondel et al., Standard model theory for the FCC-ee Tera-Z stage, in Mini Workshop on Precision EW and QCD Calculations for the FCC Studies : Methods and Techniques, , CERN Yellow Reports: Monographs Vol. 3/2019, Geneva, 2018, CERN, 1809.01830.

19. T. van Ritbergen, J. A. M. Vermaseren, and S. A. Larin, Phys. Lett. B 400, 379 (1997), hep-ph/9701390

20. M. Czakon, Nucl. Phys. B 710, 485 (2005), hep-ph/0411261

21. P. A. Baikov, K. G. Chetyrkin, and J. H. Kühn, Phys. Rev. Lett. 118, 082002 (2017), 1606.08659.

22. F. Herzog, B. Ruijl, T. Ueda, J. A. M. Vermaseren, and A. Vogt, JHEP 02, 090 (2017), 1701.01404 .

23. T. Luthe, A. Maier, P. Marquard, and Y. Schröder, JHEP 10, 166 (2017), 1709.07718.

24. J. A. M. Vermaseren, A. Vogt, and S. Moch, Nucl. Phys. B 724, 3 (2005), hep-ph/0504242

25. S. Moch, J. A. M. Vermaseren, and A. Vogt, Nucl. Phys. B 813, 220 (2009), 0812.4168.

26. B. Ruijl, T. Ueda, J. A. M. Vermaseren, J. Davies, and A. Vogt, PoS LL2016, 071 (2016), 1605.08408 .

27. G. Das, S. Moch, and A. Vogt, JHEP 03, 116 (2020), 1912.12920.

28. S. Moch, B. Ruijl, T. Ueda, J. A. M. Vermaseren, and A. Vogt, JHEP 10, 041 (2017), 1707.08315

29. S. Moch, B. Ruijl, T. Ueda, J. A. M. Vermaseren, and A. Vogt, Phys. Lett. B 782, 627 (2018), 1805.09638 .

30. J. Davies, A. Vogt, B. Ruijl, T. Ueda, and J. A. M. Vermaseren, Nucl. Phys. B 915, 335 (2017), 1610.07477.

31. F. Herzog et al., Phys. Lett. B 790, 436 (2019), 1812.11818.

32. A. J. Buras, Rev. Mod. Phys. 52, 199 (1980).

33. V. Magerya and A. Pikelner, JHEP 12, 026 (2019), 1910.07522.

34. P. Nogueira, J. Comput. Phys. 105, 279 (1993). 
35. T. van Ritbergen, A. N. Schellekens, and J. A. M. Vermaseren, Int. J. Mod. Phys. A 14, 41 (1999), hep-ph/9802376

36. G. 't Hooft and M. J. G. Veltman, Nucl. Phys. B 44, 189 (1972).

37. C. G. Bollini and J. J. Giambiagi, Nuovo Cim. B 12, 20 (1972).

38. F. V. Tkachov, Phys. Lett. B 100, 65 (1981).

39. K. G. Chetyrkin and F. V. Tkachov, Nucl. Phys. B 192, 159 (1981).

40. B. Ruijl, T. Ueda, and J. A. M. Vermaseren, Comput. Phys. Commun. 253, 107198 (2020), 1704.06650.

41. P. A. Baikov and K. G. Chetyrkin, Nucl. Phys. B 837, 186 (2010), 1004.1153.

42. R. N. Lee, A. V. Smirnov, and V. A. Smirnov, Nucl. Phys. B 856, 95 (2012), 1108.0732.

43. J. A. M. Vermaseren, (2000), math-ph/0010025

44. J. Kuipers, T. Ueda, J. A. M. Vermaseren, and J. Vollinga, Comput. Phys. Commun. 184, 1453 (2013), 1203.6543.

45. B. Ruijl, T. Ueda, and J. Vermaseren, (2017), 1707.06453.

46. M. Tentyukov and J. A. M. Vermaseren, Comput. Phys. Commun. 181, 1419 (2010), hep$\mathrm{ph} / 0702279$

47. V. N. Velizhanin, Nucl. Phys. B 864, 113 (2012), 1203.1022.

48. J. A. M. Vermaseren, Int. J. Mod. Phys. A 14, 2037 (1999), hep-ph/9806280

49. J. Blümlein and S. Kurth, Phys. Rev. D 60, 014018 (1999), hep-ph/9810241

50. A. K. Lenstra, H. W. Lenstra, and L. Lovász, Mathematische Annalen 261, 515 (1982)

51. K. Matthews, (unpublished), summarized in [52]; see pp. 16/17 .

52. J. H. Silverman, Des. Codes Cryptography 20, 5 (2000).

53. http://www. numbertheory.org/calc/krm_calc.html

54. F. Herzog and B. Ruijl, JHEP 05, 037 (2017), 1703.03776.

55. K. G. Chetyrkin and F. V. Tkachov, Phys. Lett. B 114, 340 (1982).

56. K. G. Chetyrkin and V. A. Smirnov, Phys. Lett. B 144, 419 (1984)

57. K. G. Chetyrkin, (2017), 1701.08627.

58. P. Nason and B. R. Webber, Nucl. Phys. B 421, 473 (1994), [Erratum: Nucl.Phys.B 480, 755 (1996)].

59. A. Gehrmann-De Ridder, T. Gehrmann, and G. Heinrich, Nucl. Phys. B 682, 265 (2004), hep-ph/0311276

60. C. Anastasiou and K. Melnikov, Nucl. Phys. B 646, 220 (2002), hep-ph/0207004

61. O. Gituliar, V. Magerya, and A. Pikelner, JHEP 06, 099 (2018), 1803.09084.

62. G. Heinrich, T. Huber, and D. Maitre, Phys. Lett. B 662, 344 (2008), 0711.3590.

63. G. Heinrich, T. Huber, D. A. Kosower, and V. A. Smirnov, Phys. Lett. B 678, 359 (2009), 0902.3512

64. R. N. Lee, A. V. Smirnov, and V. A. Smirnov, JHEP 04, 020 (2010), 1001.2887.

65. O. V. Tarasov, Phys. Rev. D 54, 6479 (1996), hep-th/9606018

66. O. V. Tarasov, Nucl. Phys. B Proc. Suppl. 89, 237 (2000), hep-ph/0102271

67. R. N. Lee, Nucl. Phys. B 830, 474 (2010), 0911.0252.

68. R. N. Lee and K. T. Mingulov, (2017), 1712.05173.

69. J. Blümlein, D. J. Broadhurst, and J. A. M. Vermaseren, Comput. Phys. Commun. 181, 582 (2010), 0907.2557.

70. H. R. P. Ferguson, D. H. Bailey, and S. Arno, Math. Comput. 68, 351 (1999).

71. R. E. Cutkosky, J. Math. Phys. 1, 429 (1960).

72. G. 't Hooft and M. J. G. Veltman, NATO Sci. Ser. B 4, 177 (1974).

73. O. Gituliar, JHEP 02, 017 (2016), 1512.02045.

74. O. Gituliar and S. Moch, Acta Phys. Polon. B 46, 1279 (2015), 1505.02901.

75. V. Magerya, (2021), Ph.D.thesis (Universität Hamburg).

76. A. V. Kotikov, Phys. Lett. B 254, 158 (1991).

77. A. V. Kotikov, Phys. Lett. B 267, 123 (1991), [Erratum: Phys.Lett.B 295, 409-409 (1992)].

78. J. M. Henn, Phys. Rev. Lett. 110, 251601 (2013), 1304.1806.

79. R. N. Lee, JHEP 04, 108 (2015), 1411.0911.

80. R. N. Lee and A. A. Pomeransky, (2017), 1707.07856.

81. O. Gituliar and V. Magerya, Comput. Phys. Commun. 219, 329 (2017), 1701.04269.

82. O. Gituliar and V. Magerya, PoS LL2016, 030 (2016), 1607.00759.

83. https://github.com/magv/fuchsia.cpp. 